Article

\title{
Some New q-Congruences for Truncated Basic Hypergeometric Series
}

\author{
Victor J. W. Guo ${ }^{1}$ and Michael J. Schlosser ${ }^{2, *}$ (1) \\ 1 School of Mathematical Sciences, Huaiyin Normal University, Huai'an 223300, China; jwguo@hytc.edu.cn \\ 2 Fakultät für Mathematik, Universität Wien, Oskar-Morgenstern-Platz 1, A-1090 Vienna, Austria \\ * Correspondence: michael.schlosser@univie.ac.at
}

Received: 27 January 2019; Accepted: 18 February 2019; Published: 20 February 2019

check for updates

\begin{abstract}
We provide several new $q$-congruences for truncated basic hypergeometric series, mostly of arbitrary order. Our results include congruences modulo the square or the cube of a cyclotomic polynomial, and in some instances, parametric generalizations thereof. These are established by a variety of techniques including polynomial argument, creative microscoping (a method recently introduced by the first author in collaboration with Zudilin), Andrews' multiseries generalization of the Watson transformation, and induction. We also give a number of related conjectures including congruences modulo the fourth power of a cyclotomic polynomial.
\end{abstract}

Keywords: basic hypergeometric series; supercongruences; $q$-congruences; cyclotomic polynomial; Andrews' transformation; $q$-binomial theorem

MSC: 11A07, 11F33, 33D15

\section{Introduction}

In 1914, Ramanujan [1] stated rather mysteriously a number of formulas for $1 / \pi$, including

$$
\sum_{k=0}^{\infty} \frac{\left(\frac{1}{2}\right)_{k}^{3}}{k !^{3}}(6 k+1) \frac{1}{4^{k}}=\frac{4}{\pi}
$$

In 1997, Van Hamme [2] conjectured 13 interesting p-adic analogues of Ramanujan's or Ramanujan-type formulas for $1 / \pi$, such as

$$
\sum_{k=0}^{\frac{p-1}{2}} \frac{\left(\frac{1}{2}\right)_{k}^{3}}{k !^{3}}(6 k+1) \frac{1}{4^{k}} \equiv p(-1)^{\frac{p-1}{2}} \quad\left(\bmod p^{4}\right),
$$

where $(a)_{n}=a(a+1) \cdots(a+n-1)$ denotes the Pochhammer symbol and $p$ is an odd prime. All of the 13 supercongruences have been confirmed by different techniques up to now (see [3,4]). For some informative background on Ramanujan-type supercongruences, see Zudilin's paper [5]. During the past few years, $q$-analogues of congruences and supercongruences have caught the interests of many authors (see, for example, [6-28]). As made explicit in [22], $q$-supercongruences are related to studying the asymptotic behaviour of $q$-series at roots of unity. This hints towards an intrinsic connection to mock theta functions and quantum modular forms (see, e.g., [29,30]).

Congruences of truncated hypergeometric series modulo a high power of a prime such as in Equation (1) are special. Similarly, in the setting of truncated basic hypergeometric series, congruences modulo some power of a cyclotomic polynomial are special and, already for the exponent being $\geqslant 2$, are typically difficult to prove. 
Recently, the first author (Theorem 1.1, [28]) proved that for $n \equiv 4(\bmod 5)$

$$
\sum_{k=0}^{n-1} \frac{\left(q ; q^{5}\right)_{k}^{5}}{\left(q^{5} ; q^{5}\right)_{k}^{5}} q^{5 k} \equiv 0 \quad\left(\bmod \Phi_{n}(q)^{2}\right)
$$

which, under the substitution $q \mapsto q^{-1}$, can be written as

$$
\sum_{k=0}^{n-1} \frac{\left(q ; q^{5}\right)_{k}^{5}}{\left(q^{5} ; q^{5}\right)_{k}^{5}} q^{15 k} \equiv 0 \quad\left(\bmod \Phi_{n}(q)^{2}\right)
$$

It follows that for $n \equiv 4(\bmod 5)$

$$
\sum_{k=0}^{n-1}[10 k+1] \frac{\left(q ; q^{5}\right)_{k}^{5}}{\left(q^{5} ; q^{5}\right)_{k}^{5}} q^{5 k} \equiv 0 \quad\left(\bmod \Phi_{n}(q)^{2}\right)
$$

Here and in what follows, we adopt the standard $q$-notation: $(a ; q)_{n}=(1-a)(1-a q) \cdots\left(1-a q^{n-1}\right)$ is the $q$-shifted factorial; $\left(a_{1}, a_{2}, \ldots, a_{m} ; q\right)_{n}=\left(a_{1} ; q\right)_{n}\left(a_{2} ; q\right)_{n} \cdots\left(a_{m} ; q\right)_{n}$ is a product of $q$-shifted factorials; $[n]=[n]_{q}=1+q+\cdots+q^{n-1}$ is the $q$-integer; and $\Phi_{n}(q)$ denotes the $n$-th cyclotomic polynomial in $q$ (see [31]), which may be defined as

$$
\Phi_{n}(q)=\prod_{\substack{1 \leqslant k \leqslant n \\ \operatorname{gcd}(n, k)=1}}\left(q-\zeta^{k}\right),
$$

where $\zeta$ is an $n$-th primitive root of unity.

We find that for $n \equiv 2(\bmod 5)$ the $q$-congruence (2) even holds modulo $\Phi_{n}(q)^{3}$. More generally we are able to extend (2) to the following infinite family of $q$-congruences.

Theorem 1. Let $d \geqslant 5$ be an odd integer. Then

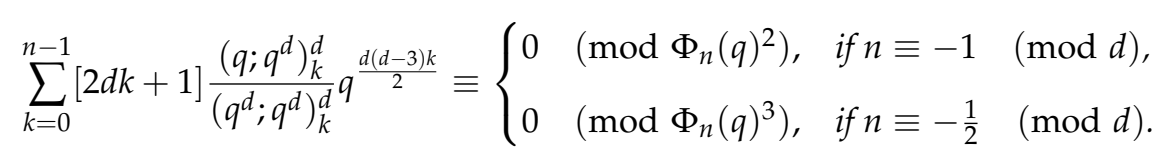

Note that for $d \geqslant 7$ and $n \equiv-1(\bmod d)$ the above $q$-congruence cannot be deduced directly from [28] (Theorem 1.1) in the same way as the $q$-congruence (2) is derived. This is because the arguments $q^{d}$ and $q^{\frac{d(d-3)}{2}}$ are different for $d \geqslant 7$. It should be pointed out that the $q$-congruence (3) does not hold for $d=3$. Like many results given in [24], Theorem 1 has a companion as follows.

Theorem 2. Let $d \geqslant 3$ be an odd integer and let $n>1$. Then

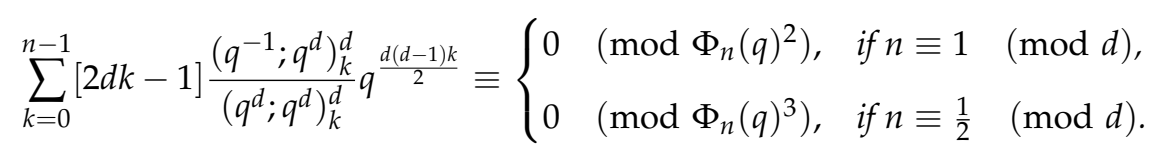

We shall also prove the following result, which was originally conjectured by the first author [28] (Conjecture 1.3) who provided a proof of the modulus $[n] \Phi_{n}(q)$ case [28] (Theorem 1.2).

Theorem 3. Let $n>1$ be an odd integer. Then

$$
\sum_{k=0}^{n-1} \frac{\left(q^{-1} ; q^{2}\right)_{k}^{2}}{\left(q^{2} ; q^{2}\right)_{k}^{2}} q^{2 k} \equiv 0 \quad\left(\bmod [n]^{2}\right)
$$


and

$$
\sum_{k=0}^{\frac{n+1}{2}} \frac{\left(q^{-1} ; q^{2}\right)_{k}^{2}}{\left(q^{2} ; q^{2}\right)_{k}^{2}} q^{2 k} \equiv 0 \quad\left(\bmod [n]^{2}\right)
$$

As mentioned in [28], there are many similar congruences modulo $\Phi_{n}(q)^{2}$ for truncated basic hypergeometric series. In this paper, we shall give more such examples (theorems or conjectures). The simplest example is as follows.

Theorem 4. Let $n \geqslant 4$ be an integer with $\operatorname{gcd}(n, 3)=1$. Then

$$
\sum_{k=0}^{n-1} \frac{\left(q^{-1}, q^{-2} ; q^{3}\right)_{k}}{\left(q^{3} ; q^{3}\right)_{k}^{2}} q^{3 k} \equiv 0 \quad\left(\bmod \Phi_{n}(q)^{2}\right) .
$$

Note that the first author, in joint work with Pan and Zhang [11], proved that for any odd integer $n \geqslant 5$ with $\operatorname{gcd}(n, 3)=1$ there holds

$$
\sum_{k=0}^{n-1} \frac{\left(q, q^{2} ; q^{3}\right)_{k}}{\left(q^{3} ; q^{3}\right)_{k}^{2}} q^{3 k} \equiv\left(\frac{n}{3}\right) q^{\frac{n^{2}-1}{3}} \quad\left(\bmod \Phi_{n}(q)^{2}\right)
$$

where $(\dot{\overline{3}})$ denotes the Legendre symbol modulo 3 . This $q$-congruence was originally conjectured in [10] when $n=p$ is an odd prime.

We shall prove Theorems 1 and 2 in Sections 2 and 3 by using the creative microscoping method developed by the first author and Zudilin [22]. We prove these by first establishing their parametric generalizations modulo $\left(1-a q^{n}\right)\left(a-q^{n}\right)$ and then letting $a \rightarrow 1$. The proofs are similar to that of [28] (Theorem 1.1) but also require Andrews' multiseries generalization of the Watson transformation [32] (Theorem 4) (which was already used by the first author, Jouhet and Zeng [6], for proving some $q$-analogues of Calkin's congruence [33]). It is worth mentioning that we need to add the parameter $a$ and also its powers in many places of the left-hand sides of Equations (3) and (4) in order to establish the desired generalizations modulo $\left(1-a q^{n}\right)\left(a-q^{n}\right)$. Therefore, the proofs of Theorems 1 and 2 are quite different from those in the recent two joint papers of us $[24,25]$, where the parameter $a$ is inserted in a more natural way (without $a^{2}$ and higher powers of $a$ ) as done in [22]. The proofs of Theorems 3 and 4 are based on two $q$-series identities and are given in Sections 4 and 5, respectively. Two more congruences modulo $\Phi_{n}(q)^{2}$ are given in Section 6. We give some related conjectures in the final Section 7. These include two refinements of Theorems 1 and 2, some extensions of Theorem 4 for $n \equiv 1$ ( $\bmod 3)$, and similar conjectures.

\section{Proof of Theorem 1} $(\bmod d)$.

We first establish the following parametric generalization of Theorem 1 for the case $n \equiv-1$

Theorem 5. Let $d \geqslant 5$ be an odd integer and let $n \equiv-1(\bmod d)$. Then modulo $\left(1-a q^{n}\right)\left(a-q^{n}\right)$,

$$
\begin{aligned}
& \sum_{k=0}^{n-1}[2 d k+1] \frac{\left(a^{d-1} q, a^{d-3} q, \ldots, a^{2} q ; q^{d}\right)_{k}}{\left(a^{d-2} q^{d}, a^{d-4} q^{d}, \ldots, a q^{d} ; q^{d}\right)_{k}} \\
& \quad \times \frac{\left(a^{1-d} q, a^{3-d} q, \ldots, a^{-2} q ; q^{d}\right)_{k}\left(q ; q^{d}\right)_{k}}{\left(a^{2-d} q^{d}, a^{4-d} q^{d}, \ldots, a^{-1} q^{d} ; q^{d}\right)_{k}\left(q^{d} ; q^{d}\right)_{k}} q^{\frac{d(d-3) k}{2}} \equiv 0 .
\end{aligned}
$$


Proof. It is clear that $\operatorname{gcd}(d, n)=1$ and therefore the numbers $d, 2 d, \ldots,(n-1) d$ are all not divisible by $n$. This implies that the denominators of the left-hand side of (8) do not contain the factor $1-a q^{n}$ nor $1-a^{-1} q^{n}$. Thus, for $a=q^{-n}$ or $a=q^{n}$, the left-hand side of (8) can be written as

$$
\begin{aligned}
& \sum_{k=0}^{\frac{d n-n-1}{d}}[2 d k+1] \frac{\left(q^{1-(d-1) n}, q^{1-(d-3) n}, \ldots, q^{1-2 n} ; q^{d}\right)_{k}}{\left(q^{d-(d-2) n}, q^{d-(d-4) n}, \ldots, q^{d-n} ; q^{d}\right)_{k}} \\
& \quad \times \frac{\left(q^{(d-1) n+1}, q^{(d-3) n+1}, \ldots, q^{2 n+1} ; q^{d}\right)_{k}\left(q ; q^{d}\right)_{k}}{\left(q^{(d-2) n+d}, q^{(d-4) n+d}, \ldots, q^{n+d} ; q^{d}\right)_{k}\left(q^{d} ; q^{d}\right)_{k}} q^{\frac{d(d-3) k}{2}}
\end{aligned}
$$

where we have used $\left(q^{1-(d-1) n} ; q^{d}\right)_{k}=0$ for $k>(d n-n-1) / d$.

Let

$$
\left[\begin{array}{l}
n \\
k
\end{array}\right]=\left[\begin{array}{l}
n \\
k
\end{array}\right]_{q}=\frac{(q ; q)_{n}}{(q ; q)_{k}(q ; q)_{n-k}}
$$

be the $q$-binomial coefficient. It is easy to see, with $\left(\begin{array}{l}k \\ 2\end{array}\right)=k(k-1) / 2$ denoting a binomial coefficient, that

$$
\begin{aligned}
\frac{\left(q^{1-(d-1) n} ; q^{d}\right)_{k}}{\left(q^{d} ; q^{d}\right)_{k}} q^{\frac{d(d-3) k}{2}} & =(-1)^{k}\left[\begin{array}{c}
(d n-n-1) / d \\
k
\end{array}\right]_{q^{d}} q^{d\left(\begin{array}{l}
k \\
2
\end{array}\right)+\left(n+1-d n+\frac{d(d-3)}{2}\right) k}, \\
\frac{\left(q^{1-(d-3) n} ; q^{d}\right)_{k}}{\left(q^{d-(d-2) n} ; q^{d}\right)_{k}} & =\frac{\left(q^{d-(d-2) n+d k} ; q^{d}\right)_{(n+1-d) / d}}{\left(q^{d-(d-2) n} ; q^{d}\right)_{(n+1-d) / d}} \\
\frac{\left(q^{1-(d-5) n} ; q^{d}\right)_{k}}{\left(q^{d-(d-4) n} ; q^{d}\right)_{k}} & =\frac{\left(q^{d-(d-4) n+d k} ; q^{d}\right)_{(n+1-d) / d}}{\left(q^{d-(d-4) n} ; q^{d}\right)_{(n+1-d) / d}} \\
& \vdots \\
\frac{\left(q^{1-2 n} ; q^{d}\right)_{k}}{\left(q^{d-3 n} ; q^{d}\right)_{k}} & =\frac{\left(q^{d-3 n+d k} ; q^{d}\right)_{(n+1-d) / d}}{\left(q^{d-3 n} ; q^{d}\right)_{(n+1-d) / d}}
\end{aligned}
$$

and

$$
\begin{aligned}
\frac{\left(q^{(d-1) n+1} ; q^{d}\right)_{k}}{\left(q^{(d-2) n+d} ; q^{d}\right)_{k}} & =\frac{\left(q^{(d-2) n+d k+d} ; q^{d}\right)_{(n+1-d) / d}}{\left(q^{(d-2) n+d} ; q^{d}\right)_{(n+1-d) / d}} \\
\frac{\left(q^{(d-3) n+1} ; q^{d}\right)_{k}}{\left(q^{(d-4) n+d} ; q^{d}\right)_{k}} & =\frac{\left(q^{(d-4) n+d k+d} ; q^{d}\right)_{(n+1-d) / d}}{\left(q^{(d-4) n+d} ; q^{d}\right)_{(n+1-d) / d}} \\
& \vdots \\
\frac{\left(q^{2 n+1} ; q^{d}\right)_{k}}{\left(q^{n+d} ; q^{d}\right)_{k}} & =\frac{\left(q^{2 n+d k+d} ; q^{d}\right)_{(n+1-d) / d}}{\left(q^{n+d} ; q^{d}\right)_{(n+1-d) / d}} \\
\frac{\left(q ; q^{d}\right)_{k}}{\left(q^{d-n} ; q^{d}\right)_{k}} & =\frac{\left(q^{d-n+d k} ; q^{d}\right)_{(n+1-d) / d}}{\left(q^{d-n} ; q^{d}\right)_{(n+1-d) / d}}
\end{aligned}
$$

Note that the right-hand sides of the identities after (10) are all polynomials in $q^{d k}$, of degree $(n+1-d) / d$ in the first group, and of degree $(n+1-d) / d$ in the second one too. Moreover,

$$
d\left(\begin{array}{l}
k \\
2
\end{array}\right)+\left(n+1-d n+\frac{d(d-3)}{2}\right) k
$$




$$
=d\left(\begin{array}{c}
(d n-n-1) / d-k \\
2
\end{array}\right)-d\left(\begin{array}{c}
(d n-n-1) / d \\
2
\end{array}\right)+\frac{d(d-5) k}{2} .
$$

Therefore, we can write (9) in the following form

$$
\sum_{k=0}^{\frac{d n-n-1}{d}}(-1)^{k} q^{d\left(\begin{array}{l}
(d n-n-1) / d-k \\
2
\end{array}\right)}\left[\begin{array}{c}
(d n-n-1) / d \\
k
\end{array}\right]_{q^{d}} P\left(q^{d k}\right),
$$

where $P\left(q^{d k}\right)$ is a polynomial in $q^{d k}$ of degree $2+(n+1-d)(d-1) / d+(d-5) / 2=(d n-n-1) / d-$ $(d-3) / 2 \leqslant(d n-n-1) / d-1$.

Recall that the $q$-binomial theorem (see [34] (p. 36)) can be written as

$$
\sum_{k=0}^{n}(-1)^{k}\left[\begin{array}{l}
n \\
k
\end{array}\right] q^{\left(\frac{k}{2}\right) z^{k}}=(z ; q)_{n}
$$

Putting $z=q^{-j}$ in the above identity and replacing $k$ with $n-k$, we get

$$
\sum_{k=0}^{n}(-1)^{k}\left[\begin{array}{l}
n \\
k
\end{array}\right] q^{\left(\frac{n-k}{2}\right)+j k}=0 \quad \text { for } 0 \leqslant j \leqslant n-1,
$$

which immediately means that the expression in (9), which equals (11), vanishes. This proves (8).

In order to prove Theorem 1 for the case $n \equiv-\frac{1}{2}(\bmod d)$, we need the following lemma.

Lemma 1. Let $d \geqslant 3$ be an odd integer and let $n \equiv-\frac{1}{2}(\bmod d)$. Then for $0 \leqslant k \leqslant(d n-2 n-1) / d$, modulo $\Phi_{n}(q)$ we have

$$
\frac{\left(a q ; q^{d}\right)_{(d n-2 n-1) / d-k}}{\left(q^{d} / a ; q^{d}\right)_{(d n-2 n-1) / d-k}} \equiv(-a)^{(d n-2 n-1) / d-2 k} \frac{\left(a q ; q^{d}\right)_{k}}{\left(q^{d} / a ; q^{d}\right)_{k}} q^{(d n-2 n-d+1)(d n-2 n-1) /(2 d)+(d-1) k} .
$$

Proof. Since $q^{n} \equiv 1\left(\bmod \Phi_{n}(q)\right)$, we have

$$
\begin{aligned}
\frac{\left(a q ; q^{d}\right)_{(d n-2 n-1) / d}}{\left(q^{d} / a ; q^{d}\right)_{(d n-2 n-1) / d}} & =\frac{(1-a q)\left(1-a q^{d+1}\right) \cdots\left(1-a q^{d n-2 n-d}\right)}{\left(1-q^{d} / a\right)\left(1-q^{2 d} / a\right) \cdots\left(1-q^{d n-2 n-1 / a}\right)} \\
& \equiv \frac{(1-a q)\left(1-a q^{d+1}\right) \cdots\left(1-a q^{d n-2 n-d}\right)}{\left(1-q^{d+2 n-d n / a)\left(1-q^{2 d+2 n-d n} / a\right) \cdots\left(1-q^{-1 / a}\right)}\right.} \\
& =(-a)^{(d n-2 n-1) / d} q^{(d n-2 n-d+1)(d n-2 n-1) /(2 d)} \quad\left(\bmod \Phi_{n}(q)\right) .
\end{aligned}
$$

Furthermore, modulo $\Phi_{n}(q)$, there holds

$$
\begin{aligned}
& \frac{\left(a q ; q^{d}\right)_{(d n-2 n-1) / d-k}}{\left(q^{d} / a ; q^{d}\right)_{(d n-2 n-1) / d-k}} \\
& =\frac{\left(a q ; q^{d}\right)_{(d n-2 n-1) / d}}{\left(q^{d} / a ; q^{d}\right)_{(d n-2 n-1) / d}} \frac{\left(1-q^{d n-2 n+d-1-d k} / a\right)\left(1-q^{d n-2 n+2 d-1-d k} / a\right) \cdots\left(1-q^{d n-2 n-1} / a\right)}{\left(1-a q^{d n-2 n-d k}\right)\left(1-a q^{d n-2 n+d-d k}\right) \cdots\left(1-a q^{d n-2 n-d}\right)} \\
& \equiv \frac{\left(a q ; q^{d}\right)_{(d n-2 n-1) / d}}{\left(q^{d} / a ; q^{d}\right)_{(d n-2 n-1) / d}} \frac{\left(1-q^{d-1-d k} / a\right)\left(1-q^{2 d-1-d k} / a\right) \cdots\left(1-q^{-1} / a\right)}{\left(1-a q^{-d k}\right)\left(1-a q^{d-d k}\right) \cdots\left(1-a q^{-d}\right)}
\end{aligned}
$$

which together with (13) establishes the assertion. 
We now give a parametric generalization of Theorem 1 for the case $n \equiv-\frac{1}{2}(\bmod d)$.

Theorem 6. Let $d \geqslant 3$ be an odd integer and let $n \equiv-\frac{1}{2}(\bmod d)$. Then modulo $\Phi_{n}(q)\left(1-a q^{n}\right)\left(a-q^{n}\right)$,

$$
\begin{aligned}
& \sum_{k=0}^{n-1}[2 d k+1] \frac{\left(a^{d-2} q, a^{d-4} q, \ldots, a q ; q^{d}\right)_{k}}{\left(a^{d-2} q^{d}, a^{d-4} q^{d}, \ldots, a q^{d} ; q^{d}\right)_{k}} \\
& \quad \times \frac{\left(a^{2-d} q, a^{4-d} q, \ldots, a^{-1} q ; q^{d}\right)_{k}\left(q ; q^{d}\right)_{k}}{\left(a^{2-d} q^{d}, a^{4-d} q^{d}, \ldots, a^{-1} q^{d} ; q^{d}\right)_{k}\left(q^{d} ; q^{d}\right)_{k}} q^{\frac{d(d-3) k}{2}} \equiv 0 .
\end{aligned}
$$

Proof. By Lemma 1, for $0 \leqslant k \leqslant(d n-2 n-1)$, we can check that the $k$-th and $((d n-2 n-1) / d-k)$-th terms on the left-hand side of (14) modulo $\Phi_{n}(q)$ cancel each other. Moreover, for $(d n-2 n-1) / d<k \leqslant n-1$, the $q$-shifted factorial $\left(q ; q^{d}\right)_{k}$ contains the factor $1-q^{n}$ and is therefore divisible by $\Phi_{n}(q)$. This proves that the congruence (14) is true modulo $\Phi_{n}(q)$.

To prove that (14) is also true modulo $\left(1-a q^{n}\right)\left(a-q^{n}\right)$, it suffices to prove the following identity:

$$
\begin{aligned}
& \sum_{k=0}^{\frac{d n-2 n-1}{d}}[2 d k+1] \frac{\left(q^{1-(d-2) n}, q^{1-(d-4) n}, \ldots, q^{1-n} ; q^{d}\right)_{k}}{\left(q^{d-(d-2) n}, q^{d-(d-4) n}, \ldots, q^{d-n} ; q^{d}\right)_{k}} \\
& \quad \times \frac{\left(q^{(d-2) n+1}, q^{(d-4) n+1}, \ldots, q^{n+1} ; q^{d}\right)_{k}\left(q ; q^{d}\right)_{k}}{\left(q^{(d-2) n+d}, q^{(d-4) n+d}, \ldots, q^{n+d} ; q^{d}\right)_{k}\left(q^{d} ; q^{d}\right)_{k}} q^{\frac{d(d-3) k}{2}}=0
\end{aligned}
$$

where we have used that $\left(q^{1-(d-2) n} ; q^{d}\right)_{k}=0$ for $k>(d n-2 n-1) / d$. This time the method employed to prove (9) does not work. Instead, we shall use Andrews' multiseries generalization of the Watson transformation [32] (Theorem 4):

$$
\begin{aligned}
& \sum_{k \geqslant 0} \frac{\left(a, q \sqrt{a},-q \sqrt{a}, b_{1}, c_{1}, \ldots, b_{m}, c_{m}, q^{-N} ; q\right)_{k}}{\left(q, \sqrt{a},-\sqrt{a}, a q / b_{1}, a q / c_{1}, \ldots, a q / b_{m}, a q / c_{m}, a q^{N+1} ; q\right)_{k}}\left(\frac{a^{m} q^{m+N}}{b_{1} c_{1} \cdots b_{m} c_{m}}\right)^{k} \\
& =\frac{\left(a q, a q / b_{m} c_{m} ; q\right)_{N}}{\left(a q / b_{m}, a q / c_{m} ; q\right)_{N}} \sum_{l_{1}, \ldots, l_{m-1} \geqslant 0} \frac{\left(a q / b_{1} c_{1} ; q\right)_{l_{1}} \cdots\left(a q / b_{m-1} c_{m-1} ; q\right)_{l_{m-1}}}{(q ; q)_{l_{1}} \cdots(q ; q)_{l_{m-1}}} \\
& \times \frac{\left.\left(b_{2}, c_{2} ; q\right)_{l_{1}} \ldots\left(b_{m}, c_{m} ; q\right)\right)_{l_{1}+\cdots+l_{m-1}}}{\left(a q / b_{1}, a q / c_{1} ; q\right)_{l_{1}} \ldots\left(a q / b_{m-1}, a q / c_{m-1} ; q\right)_{l_{1}+\cdots+l_{m-1}}}
\end{aligned}
$$

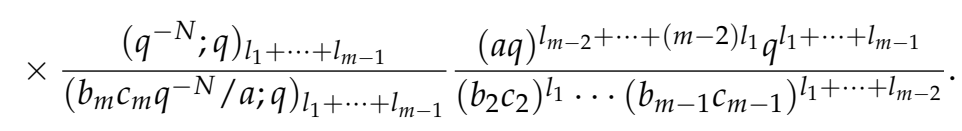

Let $q \mapsto q^{d}, a=q, b_{1}=q^{(d+1) / 2}, m=(d-1) / 2$, and $N=(d n-2 n-1) / d$ in (16). Moreover, put

$$
\left\{c_{1}, b_{2}, c_{2}, \ldots, b_{m}, c_{m}\right\}=\left\{q^{1-(d-4) n}, q^{1-(d-6) n}, \ldots, q^{1-n}, q^{(d-2) n+1}, q^{(d-4) n+1}, \ldots, q^{n+1}\right\}
$$

with $b_{m}=q^{1-n}$ and $c_{m}=q^{3 n+1}$. Then the left-hand side of (16) reduces to the left-hand side of (15), while the right-hand side of (16) contains the factor

$$
\left(q^{d+1} / b_{m} c_{m} ; q^{d}\right)_{N}=\left(q^{d-2 n-1} ; q^{d}\right)_{N}=0,
$$

because $d-2 n-1 \equiv 0(\bmod d), d-2 n-1 \leqslant 0$ and $N>(d-2 n-1) / d$. This proves (15), i.e., the congruence (14) holds modulo $\left(1-a q^{n}\right)\left(a-q^{n}\right)$. Since the polynomials $\Phi_{n}(q)$ and $\left(1-a q^{n}\right)\left(a-q^{n}\right)$ are clearly relatively prime, the proof of (14) is complete.

Proof of Theorem 1 . For $n \equiv-1(\bmod d)$, the limits of the denominators in (8) as $a \rightarrow 1$ are relatively prime to $\Phi_{n}(q)$. On the other hand, the limit of $\left(1-a q^{n}\right)\left(a-q^{n}\right)$ as $a \rightarrow 1$ has the factor $\Phi_{n}(q)^{2}$. It follows that the limiting case $a \rightarrow 1$ of the congruence (8) reduces to (3) for the case $n \equiv-1(\bmod d)$. 
Similarly, for $n \equiv-\frac{1}{2}(\bmod d)$, the limit of $\Phi_{n}(q)\left(1-a q^{n}\right)\left(a-q^{n}\right)$ as $a \rightarrow 1$ has the factor $\Phi_{n}(q)^{3}$, and so the limiting case $a \rightarrow 1$ of the congruence (14) reduces to (3) for the case $n \equiv-\frac{1}{2}(\bmod d)$. This completes the proof of the theorem.

\section{Proof of Theorem 2}

The proof of Theorem 2 is similar to that of Theorem 1. We have the following parametric generalization of Theorem 2 for the case $n \equiv-1(\bmod d)$. Its proof is completely analogous to that of Theorem 5 and is left to the interested reader.

Theorem 7. Let $d \geqslant 3$ be an odd integer and let $n \equiv 1(\bmod d)$. Then modulo $\left(1-a q^{n}\right)\left(a-q^{n}\right)$,

$$
\begin{aligned}
& \sum_{k=0}^{n-1}[2 d k-1] \frac{\left(a^{d-1} q^{-1}, a^{d-3} q^{-1}, \ldots, a^{2} q^{-1} ; q^{d}\right)_{k}}{\left(a^{d-2} q^{d}, a^{d-4} q^{d}, \ldots, a q^{d} ; q^{d}\right)_{k}} \\
& \quad \times \frac{\left(a^{1-d} q^{-1}, a^{3-d} q^{-1}, \ldots, a^{-2} q^{-1} ; q^{d}\right)_{k}\left(q^{-1} ; q^{d}\right)_{k}}{\left(a^{2-d} q^{d}, a^{4-d} q^{d}, \ldots, a^{-1} q^{d} ; q^{d}\right)_{k}\left(q^{d} ; q^{d}\right)_{k}} q^{\frac{d(d-1) k}{2}} \equiv 0 .
\end{aligned}
$$

Moreover, we have the following result similar to Lemma 1.

Lemma 2. Let $d$ be a positive odd integer and let $n \equiv \frac{1}{2}(\bmod d)$, Then for $0 \leqslant k \leqslant(d n-2 n+1) / d$, modulo $\Phi_{n}(q)$, we have

$$
\frac{\left(a q^{-1} ; q^{d}\right)_{(d n-2 n+1) / d-k}}{\left(q^{d} / a ; q^{d}\right)_{(d n-2 n+1) / d-k}} \equiv(-a)^{(d n-2 n+1) / d-2 k} \frac{\left(a q^{-1} ; q^{d}\right)_{k}}{\left(q^{d} / a ; q^{d}\right)_{k}} q^{(d n-2 n-d-1)(d n-2 n+1) /(2 d)+(d+1) k} .
$$

By Lemma 2 and Andrews' transformation (16), we can establish the following parametric generalization of Theorem 2 for the case $n \equiv \frac{1}{2}(\bmod d)$.

Theorem 8. Let $d \geqslant 3$ be an odd integer and let $n \equiv \frac{1}{2}(\bmod d)$. Then modulo $\Phi_{n}(q)\left(1-a q^{n}\right)\left(a-q^{n}\right)$,

$$
\begin{aligned}
& \sum_{k=0}^{n-1}[2 d k-1] \frac{\left(a^{d-2} q^{-1}, a^{d-4} q^{-1}, \ldots, a q^{-1} ; q^{d}\right)_{k}}{\left(a^{d-2} q^{d}, a^{d-4} q^{d}, \ldots, a q^{d} ; q^{d}\right)_{k}} \\
& \quad \times \frac{\left(a^{2-d} q^{-1}, a^{4-d} q^{-1}, \ldots, a^{-1} q^{-1} ; q^{d}\right)_{k}\left(q^{-1} ; q^{d}\right)_{k}}{\left(a^{2-d} q^{d}, a^{4-d} q^{d}, \ldots, a^{-1} q^{d} ; q^{d}\right)_{k}\left(q^{d} ; q^{d}\right)_{k}} q^{\frac{d(d-1) k}{2}} \equiv 0 .
\end{aligned}
$$

The proof of Theorem 2 then follows from Theorems 7 and 8 by taking the limit $a \rightarrow 1$.

Finally, we point out that for $d=3$ and any $n>0$ the sum in Theorem 8 has a closed form as follows:

$$
\sum_{k=0}^{n-1}[6 k-1] \frac{\left(a q^{-1}, q^{-1} / a, q^{-1} ; q^{3}\right)_{k}}{\left(a q^{3}, q^{3} / a, q^{3} ; q^{3}\right)_{k}} q^{3 k}=[3 n-2][3 n-4] \frac{\left(a q^{2}, q^{2} / a, q^{-1} ; q^{3}\right)_{n-1}}{\left(a q^{3}, q^{3} / a, q^{3} ; q^{3}\right)_{n-1}}
$$

which can be easily proved by induction on $n$. The $a=1$ case implies that when $d=3$, the congruence (4) modulo $\Phi_{n}(q)^{3}$ is still true for $n \equiv 1(\bmod 3)$ and $n>1$.

\section{Proof of Theorem 3}

By induction on $N$, we can easily prove that for $N>1$,

$$
\sum_{k=0}^{N-1} \frac{\left(q^{-1} ; q^{2}\right)_{k}^{2}}{\left(q^{2} ; q^{2}\right)_{k}^{2}} q^{2 k}=\frac{\left(q ; q^{2}\right)_{N-1}^{2}}{\left(q^{2} ; q^{2}\right)_{N-1}^{2}}\left(2[2 N-3]+q^{2 N-2}\right)
$$




$$
=\left[\begin{array}{c}
2 N-2 \\
N-1
\end{array}\right]^{2} \frac{2[2 N-3]+q^{2 N-2}}{(-q ; q)_{N-1}^{4}}
$$

Note that $\frac{1}{[N]}\left[\begin{array}{c}2 N-2 \\ N-1\end{array}\right]$ is the well-known $q$-Catalan number which is a polynomial in $q$ (see [35]). Thus $[N]$ divides $\left[\begin{array}{c}2 N-2 \\ N-1\end{array}\right]$. Moreover, it is easy to see that $[N]=\frac{1-q^{N}}{1-q}$ is relatively prime to $(-q ; q)_{N-1}$ for odd $N$. We conclude that (5) holds by taking $N=n$ in (17).

Letting $N=(n+3) / 2$ in (17), we obtain

$$
\sum_{k=0}^{\frac{n+1}{2}} \frac{\left(q^{-1} ; q^{2}\right)_{k}^{2}}{\left(q^{2} ; q^{2}\right)_{k}^{2}} q^{2 k}=\left[\begin{array}{c}
n+1 \\
(n+1) / 2
\end{array}\right]^{2} \frac{2[n]+q^{n+1}}{(-q ; q)_{(n+1) / 2}^{4}}
$$

It is clear that

$$
\frac{[(n+1) / 2]}{[n]}\left[\begin{array}{c}
n \\
(n-1) / 2
\end{array}\right]=\left[\begin{array}{c}
n-1 \\
(n-1) / 2
\end{array}\right]
$$

is a polynomial in $q$. Since the polynomials $[(n+1) / 2]$ and $[n]$ are relatively prime, we deduce that $\left[\begin{array}{c}n \\ (n-1) / 2\end{array}\right]$ is divisible by $[n]$, and so is $\left[\begin{array}{c}n+1 \\ (n+1) / 2\end{array}\right]=\left(1+q^{(n+1) / 2}\right)\left[\begin{array}{c}n \\ (n-1) / 2\end{array}\right]$. The proof of $(6)$ then follows from the fact that $[n]$ is relatively prime to $(-q ; q)_{(n+1) / 2}$.

\section{Proof of Theorem 4}

By induction on $n$, we can prove that for $n \geqslant 1$

$$
\sum_{k=0}^{n-1} \frac{\left(q^{-1}, q^{-2} ; q^{3}\right)_{k}}{\left(q^{3} ; q^{3}\right)_{k}^{2}} q^{3 k}=\frac{\left(2+q^{3 n}-q-q^{2}-q^{3 n-3}\right)\left(q, q^{2} ; q^{3}\right)_{n-1}}{(1-q)\left(1-q^{2}\right)\left(q^{3} ; q^{3}\right)_{n-1}^{2}}
$$

We now assume that $n \geqslant 4$ and $\operatorname{gcd}(n, 3)=1$. If $n \equiv 1(\bmod 3)$, then $\left(q ; q^{3}\right)_{n-1}$ contains the factor $1-q^{n}$ and $\left(q^{2} ; q^{3}\right)_{n-1}$ contains the factor $1-q^{2 n}$, and therefore $\left(q, q^{2} ; q^{3}\right)_{n-1}$ is divisible by $\Phi_{n}(q)^{2}$. If $n \equiv 2(\bmod 3)$, then $\left(q ; q^{3}\right)_{n-1}$ contains $1-q^{2 n}$ and $\left(q^{2} ; q^{3}\right)_{n-1}$ contains $1-q^{n}$, and $\left(q, q^{2} ; q^{3}\right)_{n-1}$ is also divisible by $\Phi_{n}(q)^{2}$. Clearly, the denominator of the right-hand side of (18) is relatively prime to $\Phi_{n}(q)$. This completes the proof.

\section{More Congruences Modulo $\Phi_{n}(q)^{2}$}

The first author [28] (Theorem 1.4) proved that for $n>1$,

$$
\sum_{k=0}^{n-1} \frac{\left(q, q, q^{4} ; q^{6}\right)_{k}}{\left(q^{6} ; q^{6}\right)_{k}^{3}} q^{6 k} \equiv 0 \quad\left(\bmod \Phi_{n}(q)^{2}\right) \quad \text { if } n \equiv 5 \quad(\bmod 6)
$$

and

$$
\sum_{k=0}^{n-1} \frac{\left(q^{-1}, q^{-1}, q^{-4} ; q^{6}\right)_{k}}{\left(q^{6} ; q^{6}\right)_{k}^{3}} q^{6 k} \equiv 0 \quad\left(\bmod \Phi_{n}(q)^{2}\right) \quad \text { if } n \equiv 1 \quad(\bmod 6)
$$

Here we give generalizations of the $q$-congruences (19) and (20) as follows.

Theorem 9. Let $d \geqslant 3$ and let $r$ be a nonzero integer with $|r|<d$ and $2 r \neq \pm d$. Let $n>1$ be an integer with $n \geqslant d-r$. Then

$$
\sum_{k=0}^{n-1} \frac{\left(q^{r}, q^{r}, q^{d-2 r} ; q^{d}\right)_{k}}{\left(q^{d} ; q^{d}\right)_{k}^{3}} q^{d k} \equiv 0 \quad\left(\bmod \Phi_{n}(q)^{2}\right) \quad \text { for } n \equiv-r \quad(\bmod d)
$$


Proof. The proof is similar to that of Theorem 1 (or [28] (Theorem 1.4)). Here we merely give the parametric generalization of (21):

$$
\sum_{k=0}^{n-1} \frac{\left(a^{d-1} q^{r}, a^{1-d} q^{r}, q^{d-2 r} ; q^{d}\right)_{k}}{\left(a^{d-2} q^{d}, a^{2-d} q^{d}, q^{d} ; q^{d}\right)_{k}} \equiv 0 \quad\left(\bmod \left(1-a q^{n}\right)\left(a-q^{n}\right)\right)
$$

Note that when $d=4$ and $r=1$ the $q$-congruence (21) was originally conjectured in [22] (Conjecture 5.5).

Theorem 10. Let $d \geqslant 3$ and let $0<r<d$ with $2 r \neq d$. Let $n \geqslant d+r$ be an integer. Then

$$
\sum_{k=0}^{n-1} \frac{\left(q^{-r}, q^{-r}, q^{2 r-d} ; q^{d}\right)_{k}}{\left(q^{d} ; q^{d}\right)_{k}^{3}} q^{d k} \equiv 0 \quad\left(\bmod \Phi_{n}(q)^{2}\right) \quad \text { for } n \equiv r \quad(\bmod d) .
$$

Proof. This time the parametric generalization of (22) is as follows:

$$
\sum_{k=0}^{n-1} \frac{\left(a^{d-1} q^{-r}, a^{1-d} q^{-r}, q^{2 r-d} ; q^{d}\right)_{k}}{\left(a^{d-2} q^{d}, a^{2-d} q^{d}, q^{d} ; q^{d}\right)_{k}} \equiv 0 \quad\left(\bmod \left(1-a q^{n}\right)\left(a-q^{n}\right)\right)
$$

\section{Concluding Remarks and Open Problems}

The creative microscoping method used to prove Theorems 1 and 2 can be used to prove many other $q$-congruences (see $[21,22,24,26,28]$ ). We also learned that this method has already caught the interests of Gorodetsky [23], Guillera [12] and Straub [18]. However, to the best of our knowledge, the (creative) method of adding extra parameters can only be used to prove $q$-congruences modulo $\Phi_{n}(q)^{3}$ or $\Phi_{n}(q)^{2}$ but not those modulo $\Phi_{n}(q)^{4}$ or higher powers of $\Phi_{n}(q)$. The following conjectural refinements of Theorems 1 and 2 seem to be rather challenging to prove.

Conjecture 1. Let $d \geqslant 5$ be an odd integer. Then

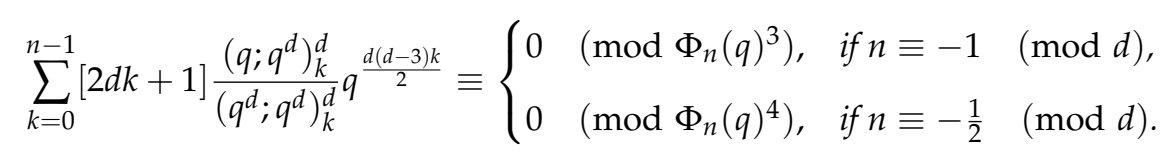

Conjecture 2. Let $d \geqslant 5$ be an odd integer and let $n>1$. Then

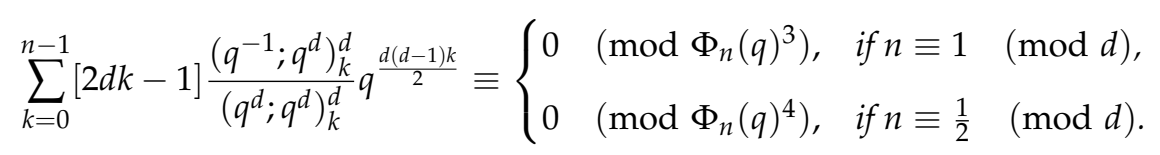

The first author ([28] (Theorem 1.1)) proved that for $d \geqslant 3$ and $n \equiv-1(\bmod d)$

$$
\sum_{k=0}^{n-1} \frac{\left(q ; q^{d}\right)_{k}^{d}}{\left(q^{d} ; q^{d}\right)_{k}^{d}} q^{d k} \equiv 0 \quad\left(\bmod \Phi_{n}(q)^{2}\right)
$$

and that for $n, d \geqslant 2$ and $n \equiv 1(\bmod d)$

$$
\sum_{k=0}^{n-1} \frac{\left(q^{-1} ; q^{d}\right)_{k}^{d}}{\left(q^{d} ; q^{d}\right)_{k}^{d}} q^{d k} \equiv 0 \quad\left(\bmod \Phi_{n}(q)^{2}\right)
$$

These two $q$-congruences were originally conjectured by the first author and Zudilin [22] (Conjectures 5.3 and 5.4). Here we would like to make some similar conjectures on congruences modulo $\Phi_{n}(q)^{2}$. 
Conjecture 3. Let $d \geqslant 3$ and $n>1$ be integers with $n \equiv-1(\bmod d(d+1) / 2)$. Then

$$
\sum_{k=0}^{n-1} \frac{\left(q, q^{2}, \ldots, q^{d} ; q^{d(d+1) / 2}\right)_{k}}{\left(q^{d(d+1) / 2} ; q^{d(d+1) / 2}\right)_{k}^{d}} q^{d(d+1) k / 2} \equiv 0 \quad\left(\bmod \Phi_{n}(q)^{2}\right) .
$$

In particular, if $p \equiv-1(\bmod d(d+1) / 2)$ is a prime and $m=d(d+1) / 2$, then

$$
\sum_{k=0}^{p-1} \frac{\left(\frac{1}{m}\right)_{k}\left(\frac{2}{m}\right)_{k} \cdots\left(\frac{d}{m}\right)_{k}}{k !^{d}} \equiv 0 \quad\left(\bmod p^{2}\right) .
$$

Conjecture 4. Let $d \geqslant 2$ and $n>1$ be integers with $n \equiv 1(\bmod d(d+1) / 2)$. Then

$$
\sum_{k=0}^{n-1} \frac{\left(q^{-1}, q^{-2}, \ldots, q^{-d} ; q^{d(d+1) / 2}\right)_{k}}{\left(q^{d(d+1) / 2} ; q^{d(d+1) / 2}\right)_{k}^{d}} q^{d(d+1) k / 2} \equiv 0 \quad\left(\bmod \Phi_{n}(q)^{2}\right) .
$$

In particular, if $p \equiv 1(\bmod d(d+1) / 2)$ is a prime and $m=d(d+1) / 2$, then

$$
\sum_{k=0}^{p-1} \frac{\left(-\frac{1}{m}\right)_{k}\left(-\frac{2}{m}\right)_{k} \cdots\left(-\frac{d}{m}\right)_{k}}{k !^{d}} \equiv 0 \quad\left(\bmod p^{2}\right)
$$

We should concede that we are not able to prove Conjectures 3 and 4 even for $d=3$ (we are only capable to deal with the modulus $\Phi_{n}(q)$ case). Note that Conjecture 4 is true for $d=2$ by Theorem 4 .

Conjecture 5. Let $d \geqslant 3$ and $n>1$ be integers with $n \equiv-1\left(\bmod d^{2}\right)$. Then

$$
\sum_{k=0}^{n-1} \frac{\left(q, q^{3}, \ldots, q^{2 d-1} ; q^{d^{2}}\right)_{k}}{\left(q^{d^{2}} ; q^{d^{2}}\right)_{k}^{d}} q^{d^{2} k} \equiv 0 \quad\left(\bmod \Phi_{n}(q)^{2}\right) .
$$

In particular, if $p \equiv-1\left(\bmod d^{2}\right)$ is a prime, then

$$
\sum_{k=0}^{p-1} \frac{\left(\frac{1}{d^{2}}\right)_{k}\left(\frac{3}{d^{2}}\right)_{k} \cdots\left(\frac{2 d-1}{d^{2}}\right)_{k}}{k !^{d}} \equiv 0 \quad\left(\bmod p^{2}\right)
$$

Conjecture 6. Let $d \geqslant 2$ and $n>1$ be integers with $n \equiv 1\left(\bmod d^{2}\right)$. Then

$$
\sum_{k=0}^{n-1} \frac{\left(q^{-1}, q^{-3}, \ldots, q^{-2 d+1} ; q^{d^{2}}\right) k}{\left(q^{d^{2}} ; q^{d^{2}}\right)_{k}^{d}} q^{d^{2} k} \equiv 0 \quad\left(\bmod \Phi_{n}(q)^{2}\right) .
$$

In particular, if $p \equiv 1\left(\bmod d^{2}\right)$ is a prime, then

$$
\sum_{k=0}^{p-1} \frac{\left(-\frac{1}{d^{2}}\right)_{k}\left(-\frac{3}{d^{2}}\right)_{k} \cdots\left(-\frac{2 d-1}{d^{2}}\right)_{k}}{k !^{d}} \equiv 0 \quad\left(\bmod p^{2}\right) .
$$

Using the following identity

$$
\sum_{k=0}^{n-1} \frac{\left(q^{-1}, q^{-3} ; q^{4}\right)_{k}}{\left(q^{4} ; q^{4}\right)_{k}^{2}} q^{4 k}=\frac{\left(2+q^{4 n}-q-q^{3}-q^{4 n-4}\right)\left(q, q^{3} ; q^{4}\right)_{n-1}}{(1-q)\left(1-q^{3}\right)\left(q^{4} ; q^{4}\right)_{n-1}^{2}}
$$

we can easily prove that Conjecture 6 is true for $d=2$.

It seems that Conjectures 3 and 4 can be further generalized as follows. 
Conjecture 7. Let $d$ and $r$ be positive integers with $d r \geqslant 3$. Let $n>1$ be an integer with $n \equiv-1$ $(\bmod d(d+1) r / 2)$. Then

$$
\sum_{k=0}^{n-1} \frac{\left(q, q^{2}, \ldots, q^{d} ; q^{d(d+1) r / 2}\right)_{k}^{r}}{\left(q^{d(d+1) r / 2} ; q^{d(d+1) r / 2}\right)_{k}^{d r}} q^{d(d+1) r k / 2} \equiv 0 \quad\left(\bmod \Phi_{n}(q)^{2}\right)
$$

Conjecture 8. Let $d$ and $r$ be positive integers with $d r \geqslant 2$. Let $n>1$ be an integer with $n \equiv 1(\bmod d(d+1) r / 2)$. Then

$$
\sum_{k=0}^{n-1} \frac{\left(q^{-1}, q^{-2}, \ldots, q^{-d} ; q^{d(d+1) r / 2}\right)_{k}^{r}}{\left(q^{d(d+1) r / 2} ; q^{d(d+1) r / 2}\right)_{k}^{d r}} q^{d(d+1) r k / 2} \equiv 0 \quad\left(\bmod \Phi_{n}(q)^{2}\right) .
$$

Likewise, Conjectures 5 and 6 can be further generalized as follows.

Conjecture 9. Let $d$ and $r$ be positive integers with $d r \geqslant 3$. Let $n>1$ be an integer with $n \equiv-1$ $\left(\bmod d^{2} r\right)$. Then

$$
\sum_{k=0}^{n-1} \frac{\left(q, q^{3}, \ldots, q^{2 d-1} ; q^{d^{2} r}\right)_{k}^{r}}{\left(q^{d^{2} r} ; q^{d^{2} r}\right)_{k}^{d r}} q^{d^{2} r k} \equiv 0 \quad\left(\bmod \Phi_{n}(q)^{2}\right)
$$

Conjecture 10. Let $d$ and $r$ be positive integers with $d r \geqslant 2$. Let $n>1$ be an integer with $n \equiv 1$ $\left(\bmod d^{2} r\right)$. Then

$$
\sum_{k=0}^{n-1} \frac{\left(q^{-1}, q^{-3}, \ldots, q^{-2 d+1} ; q^{d^{2} r}\right)_{k}^{r}}{\left(q^{d^{2} r} ; q^{d^{2} r}\right)_{k}^{d r}} q^{d^{2} r k} \equiv 0 \quad\left(\bmod \Phi_{n}(q)^{2}\right) .
$$

Finally, we point out that Conjectures 7-10 are clearly true for $d=1$ by the $d \mapsto r$ cases of (23) and (24).

Author Contributions: Both authors contributed equally.

Funding: The first author was partially supported by the National Natural Science Foundation of China (grant 11771175).

Acknowledgments: We thank Wadim Zudilin for helpful comments on a previous version of this paper. We further thank the referees for their careful reading of the manuscript; their comments led to improvements of the exposition.

Conflicts of Interest: The authors declare no conflict of interest.

\section{References}

1. Ramanujan, S. Modular equations and approximations to $\pi$. Quart. J. Math. Oxford Ser. (2) 1914, 45, 350-372.

2. Van Hamme, L. Some conjectures concerning partial sums of generalized hypergeometric series. Lectures Notes in Pure Appl. Math. 1997, 192, 223-236.

3. Swisher, H. On the supercongruence conjectures of van Hamme. Res. Math. Sci. 2015, 2, 18. [CrossRef]

4. Osburn, R.; Zudilin, W. On the (K.2) supercongruence of Van Hamme. J. Math. Anal. Appl. 2016, 433, 706-711. [CrossRef]

5. Zudilin, W. Ramanujan-type supercongruences. J. Number Theory 2009, 129, 8, 1848-1857. [CrossRef]

6. Guo, V.J.W.; Jouhet, F.; Zeng, J. Factors of alternating sums of products of binomial and $q$-binomial coefficients. Acta Arith. 2007, 127, 17-31. [CrossRef]

7. Tauraso, R. q-Analogs of some congruences involving Catalan numbers. Adv. Appl. Math. 2009, 48, 603-614. [CrossRef]

8. Pan, H.; Sun, Z.-W. Some q-congruences related to 3-adic valuations. Adv. Appl. Math. 2012, 49, $263-270$. [CrossRef]

9. Tauraso, R. Some $q$-analogs of congruences for central binomial sums. Colloq. Math. 2013, 133, $133-143$. [CrossRef] 
10. Guo, V.J.W.; Zeng, J. Some $q$-analogues of supercongruences of Rodriguez-Villegas. J. Number Theory 2014, 145, 301-316. [CrossRef]

11. Guo, V.J.W.; Pan, H.; Zhang, Y. The Rodriguez-Villegas type congruences for truncated $q$-hypergeometric functions. J. Number Theory 2017, 174, 358-368. [CrossRef]

12. Guillera, J. WZ pairs and $q$-analogues of Ramanujan series for $1 / \pi$. J. Diff. Equ. Appl. 2018, 24, 1871-1879. [CrossRef]

13. Guo, V.J.W. A $q$-analogue of a Ramanujan-type supercongruence involving central binomial coefficients. J. Math. Anal. Appl. 2018, 458, 590-600. [CrossRef]

14. Guo, V.J.W. A $q$-analogue of the (I.2) supercongruence of Van Hamme. Int. J. Number Theory 2019, 15, $29-36$. [CrossRef]

15. Guo, V.J.W. Proof of a $q$-congruence conjectured by Tauraso. Int. J. Number Theory 2019, 15, 37-41. [CrossRef]

16. Ni, H.-X.; Pan, H. On a conjectured q-congruence of Guo and Zeng. Int. J. Number Theory 2018, 14, $1699-1707$. [CrossRef]

17. Guo, V.J.W.; Wang, S.-D. Factors of sums and alternating sums of products of $q$-binomial coefficients and powers of $q$-integers. Taiwan. J. Math. 2019, $23,11-27$. [CrossRef]

18. Straub, A. Supercongruences for polynomial analogs of the Apéry numbers. Proc. Amer. Math. Soc. 2019, 147, 1023-1036. [CrossRef]

19. Guo, V.J.W. q-Analogues of the (E.2) and (F.2) supercongruences of Van Hamme. Ramanujan J. 2018. [CrossRef]

20. Guo, V.J.W. A q-analogue of a curious supercongruence of Guillera and Zudilin. J. Diff. Equ. Appl. 2019. [CrossRef]

21. Guo, V.J.W. Some q-congruences with parameters. Acta Arith. 2018, arXiv:1804.10963.

22. Guo, V.J.W.; Zudilin, W. A q-microscope for supercongruences. Adv. Math. 2019, 346, 329-358. [CrossRef]

23. Gorodetsky, O. q-Congruences, with applications to supercongruences and the cyclic sieving phenomenon. arXiv 2018, arXiv:1805.01254v1.

24. Guo, V.J.W.; Schlosser, M.J. Some $q$-supercongruences from transformation formulas for basic hypergeometric series. arXiv 2018, arXiv:1812.06324.

25. Guo, V.J.W.; Schlosser, M.J. Proof of a basic hypergeometric supercongruence modulo the fifth power of a cyclotomic polynomial. arXiv 2018, arXiv:1812.11659.

26. Guo, V.J.W.; Zudilin, W. On a q-deformation of modular forms. arXiv 2018, arXiv:1812.11322.

27. Zudilin, W. Congruences for $q$-binomial coefficients. arXiv 2019, arXiv:1901.07843.

28. Guo, V.J.W. Factors of some truncated basic hypergeometric series. arXiv 2019, arXiv:1901.07908.

29. Zagier, D. Quantum modular forms. Clay Math. Proc. 2010, 11, 659-675.

30. Folsom, A.; Ono, K.; Rhoades, R.C. Mock theta functions and quantum modular forms. Forum Math. Pi 2013, 1 e2, 27pp. [CrossRef]

31. Lang, S. Algebra. In Graduate Texts in Mathematics, 211, 3rd ed.; Axler, S., Gehring, F.W., Ribet, K.A., Eds.; Springer: New York, NY, USA, 2002.

32. Andrews, G.E. Problems and Prospects for Basic Hypergeometric Functions. In Theory and Application for Basic Hypergeometric Functions; Proceedings of an Advanced Seminar Sponsored by the Mathematics Research Center, the University of Wisconsin-Madison, March 31-April 2, 1975, Publ. No. 35; Askey, R.A., Ed.; Academic Press: New York, NY, USA, 1975, pp. 191-224.

33. Calkin, N.J. Factors of sums of powers of binomial coefficients. Acta Arith. 1998, 86, 17-26. [CrossRef]

34. Andrews, G.E. The Theory of Partitions; Cambridge University Press: Cambridge, UK, 1998.

35. Fürlinger, J.; Hofbauer, J. q-Catalan numbers. J. Combin. Theory Ser. A 1985, 2, 248-264. [CrossRef]

(c) 2019 by the authors. Licensee MDPI, Basel, Switzerland. This article is an open access article distributed under the terms and conditions of the Creative Commons Attribution (CC BY) license (http:/ / creativecommons.org/licenses/by/4.0/). 